\title{
Aprendizaje cooperativo en Educación Física
}

\author{
Carlos Velázquez Callado* \\ Antonio Fraile Aranda** \\ Víctor Manuel López Pastor ${ }^{* * *}$
}

\begin{abstract}
Resumen: La finalidad del estudio es conocer y analizar la concepción que tienen del aprendizaje cooperativo (AC) un grupo de docentes de Educación Física españoles que son reconocidos como referentes en esta metodología, así como el modo en que lo implementan en sus clases. Se trata de un estudio de caso múltiple, a través de entrevistas, observación no participante y análisis documental. Los resultados indican que todos los docentes participantes conocen los principios generales del AC y lo utilizan, lo conciben como una importante metodología para avanzar en diferentes ámbitos de aprendizaje, consideran que cualquier contenido puede ser enseñado con AC y valoran la importancia de la evaluación formativa y compartida.
\end{abstract}

Palabras clave: aprendizaje. Evaluación. Investigación cualitativa.

\section{INTRODUCCIÓN}

El aprendizaje cooperativo (AC) es definido como una metodología educativa basada en el trabajo en pequeños grupos, generalmente heterogéneos, en los que los estudiantes trabajan juntos para mejorar su propio aprendizaje y el de los demás miembros de su grupo (JOHNSON; JOHNSON; HOLUBEC, 1999). De acuerdo con el enfoque conceptual (JOHNSON; JOHNSON, 1999), el AC implica la presencia, durante el trabajo en grupo, de cinco características esenciales: (1) interdependencia positiva de metas,

\footnotetext{
'Departamento de Didáctica de la Expresión Musical, Plástica y Corporal. Facultad de Educación y Trabajo Social. Universidad de Valladolid, Valladolid, España. E-mail: carlosvelazquezcallado@gmail.com

"Departamento de Didáctica de la Expresión Musical, Plástica y Corporal. Facultad de Educación y Trabajo Social. Universidad de Valladolid, Valladolid, España. E-mail: afraile@mpc.uva.es

"'Departamento de Didáctica de la Expresión Musical, Plástica y Corporal. Escuela de Magisterio de Segovia. Universidad de Valladolid, Valladolid, España. E-mail: vlopez@mpc.uva.es
} 
que puede ser complementada con otras, como las de recursos, roles o identidad, (2) interacción promotora cara a cara, (3) responsabilidad individual, lo que implica que nadie pueda adoptar una actitud pasiva, escudándose en el trabajo de otras personas, (4) habilidades interpersonales y de trabajo en pequeño grupo y (5) procesamiento grupal o proceso mediante el cual el grupo identifica las conductas manifestadas durante el desarrollo de la tarea, determinando cuáles contribuyeron al logro de la misma y cuáles resultaron perjudiciales, con el fin de reforzar las primeras y plantear alternativas a las segundas. Actualmente, el AC es considerado una importante estrategia de enseñanza-aprendizaje, que promueve logros a nivel académico, social y afectivo-motivacional en todos los estudiantes, incluyendo aquellos con necesidades educativas específicas (GILLIES, 2006).

\section{El aprendizaje cooperativo en Educación Física (EF)}

Metzler (2011) considera ocho modelos de instrucción aplicables en las clases actuales de EF: instrucción directa, enseñanza individualizada, enseñanza recíproca, enseñanza por descubrimiento, educación deportiva, juegos tácticos, enseñanza de responsabilidad personal y social, y AC. El elemento definitorio de este último es que los estudiantes trabajan en pequeños grupos para aprender con, de y para sus compañeros.

El AC siempre implica trabajo grupal. Ahora bien, este trabajo grupal debe estar estructurado meticulosamente con el fin de que todos los estudiantes interactúen para intercambiar información, de modo que, finalmente, puedan ser evaluados de forma individual por su trabajo (FATHMAN; KESSLER, 1992). En definitiva, lo que identifica al AC, lo que le distingue del simple trabajo grupal, es la corresponsabilidad de cada estudiante por su propio aprendizaje, pero también y muy especialmente, por el de todos y cada uno de sus compañeros de grupo.

Diferentes autores destacan las ventajas de implementar el AC en las clases de EF para promover el aprendizaje y el rendimiento 
motor (ANDRÉ, 2012; BÄHR, 2010; BARRETT, 2005; CASEY, 2010), desarrollar habilidades sociales y mejorar las relaciones entre el alumnado (DYSON, 2001; FERNÁNDEZ-RÍO, 2003; GOUDAS; MAGOTSIOU, 2009; POLVI ; TELAMA, 2000), favorecer la inclusión del alumnado con discapacidad (ANDRÉ; DENEUVE; LOUVET, 2011; DOWLER, 2012; GRENIER; DYSON; YEATON, 2005; VELÁZQUEZ, 2012a), mejorar el autoconcepto general y físico (FERNÁNDEZ-RÍO, 2003) y motivar a los estudiantes hacia la práctica motriz (BARBA, 2010).

$\mathrm{El}$ análisis de la investigación sobre el $\mathrm{AC}$ en $\mathrm{EF}$ nos revela que la mayor parte de los estudios se orientan a comparar la efectividad de esta metodología en relación a otras formas de estructurar el aprendizaje (ANDRÉ, 2012; FERNÁNDEZ-RÍO, 2003) o a analizar la eficacia del AC para alcanzar diferentes objetivos motores, sociales o afectivo-motivacionales (DYSON, 2001; DOWLER, 2012; GOUDAS; MAGOTSIOU, 2009). Sin embargo, encontramos una escasez de investigaciones orientadas a determinar qué condiciones facilitan esos resultados en la práctica docente real y, en consecuencia, sobre qué variables influir para maximizar dichos logros. Dicho con otras palabras, conocemos muy poco acerca del modo en que los docentes de $\mathrm{EF}$ concretan los principios teóricos del $\mathrm{AC}$ en su práctica diaria, lo que realmente hacen cuando aplican esta metodología en sus clases.

En este sentido, planteamos un estudio dirigido a conocer y analizar la concepción que los docentes de EF españoles tienen del $\mathrm{AC}$ y el modo en que lo implementan en sus clases.

\section{Método}

Para responder al objeto de estudio hemos planteado un estudio de caso múltiple, orientado a analizar y conocer las formas de concreción del AC en las clases de EF implementadas por los docentes españoles, identificando aspectos comunes y diferencias entre teoría y práctica. 


\subsection{PARTICIPANTES}

En el estudio participaron siete docentes de EF españoles, seleccionados según los siguientes criterios: (1) haber sido identificados por otros docentes como referentes del AC en EF; (2) haber impartido clase de EF en Educación Primaria o Secundaria en los tres últimos cursos; (3) haber publicado sobre la implementación del AC en sus clases o presentado estudios en cursos de formación permanente o en congresos de EF; (4) haber manifestado que utilizan el AC en sus clases; y, (5) estar dispuestos a colaborar en nuestra investigación. Las implicaciones derivadas de esta colaboración se explicitaron a través de un acuerdo entre el investigador y cada uno de los docentes.

Tres de los docentes seleccionados impartían clase en Educación Primaria, dos en Secundaria y otros dos en la Universidad.

\subsection{RECOGIDA DE DATOS}

Las principales fuentes de recopilación de datos en este estudio fueron; (a) entrevistas con cada uno de los docentes; (b) observación no participante de diferentes clases impartidas por aquellos docentes de Educación Primaria o Secundaria durante la investigación; y (c) análisis documental (programaciones, publicaciones del docente, cuaderno del profesor, trabajos del alumnado, etc.).

El punto de partida para la recogida de datos fue una entrevista semiestructurada focalizada (MERTON; FISKE; KENDALL, 1988) o monotemática (RUIZ-OLABUÉNAGA, 2012). Dicha entrevista tenía como base un guion previo validado, diseñado a partir de la literatura sobre AC y de las aportaciones de diez expertos internacionales en esta metodología, tanto a nivel general como en $\mathrm{EF}$, a los que se les pidió que formularan diez preguntas que, a su juicio, deberían hacerse a un docente que aplicara el AC en sus clases para saber qué es lo que hace y cómo lo hace. Un primer borrador de la entrevista fue sometido a una valoración de expertos, quienes hicieron indicaciones sobre el contenido y estructura de la 
entrevista, tras lo cual se redactó el guion definitivo. El siguiente paso fue la realización de una entrevista de prueba a un docente de EF que no estaba entre los casos seleccionados, pero del que teníamos constancia de que aplicaba el AC en sus clases. El docente confirmó que la entrevista le parecía adecuada, tanto en contenido como en estructura y duración. A la entrevista principal siguieron otras complementarias, orientadas a matizar, ampliar o completar los datos obtenidos en la primera entrevista y, en el caso de los docentes de Primaria o Secundaria, de las observaciones de sus clases. Todas las entrevistas fueron grabadas y transcritas en su totalidad.

Una segunda fuente de información fue la observación no participante de un total de 24 clases de todos aquellos docentes que, durante la investigación, trabajaban en Educación Primaria o Secundaria. Las observaciones realizadas pretendían: (1) identificar en el contexto habitual de clase algunos de los hechos expuestos por el docente en las entrevistas, corroborando datos o captando posibles contradicciones, y (2) prestar atención a otros hechos o situaciones que, sin haberse manifestado en la entrevista, pudieran ser de interés para abrir nuevas preguntas acerca del modo en el que el docente aplica el AC en sus clases.

La tercera fuente de información fue el análisis documental, centrado en las programaciones de clase de los docentes, documentos oficiales del centro educativo en el que trabajaban, publicaciones de los docentes sobre el AC y, muy especialmente, materiales de apoyo utilizados por el alumnado cuando trabajaba con AC en las clases.

\subsection{ANÁLISIS DE LOS DATOS}

Para el análisis de los datos, partimos de la definición de un conjunto de categorías iniciales, derivadas del estudio previo de la literatura sobre el AC. Apoyándonos en el programa "Atlas.ti.6.2", se analizaron la totalidad de las transcripciones de las entrevistas y de las notas de campo realizadas durante las observaciones de las clases. Los datos se organizaron en un conjunto de registros 
manejables que se integraron en alguna de las categorías establecidas (DENZIN; LINCOLN, 2005). Cuando esto no era posible, se definía una nueva categoría de análisis, una categoría emergente.

Siguiendo el modelo de Huberman y Miles (1994), el siguiente paso fue reducir categorías, agrupando en una única todas aquellas que tenían un elemento identificador común. De este modo, al final de dicho proceso definimos dos grandes núcleos temáticos organizadores de toda la información: concepción del AC y aplicación del AC.

Los datos obtenidos del análisis documental se integraron en los núcleos temáticos definidos, permitiéndonos así la triangulación de fuentes de datos, lo que contribuyó a fortalecer la fiabilidad de nuestras interpretaciones.

\subsection{INDICADORES DE RIGOR}

De acuerdo con Guba (1989), se consideraron cuatro criterios para garantizar el rigor de nuestra investigación: credibilidad, transferibilidad, dependencia y confirmabilidad. Para ello, se plantearon distintas estrategias orientadas a favorecer la presencia de dichos criterios en nuestro estudio de corte cualitativo, entre las que destacamos: la consulta a expertos, la comprobación de datos y resultados con los docentes participantes en la investigación, la recogida de material referencial, la triangulación de personas y técnicas de recogida de datos, y la inclusión de descriptores de baja inferencia.

\section{Resultados}

Los resultados del estudio sobre la concepción de los docentes de EF acerca del AC y el modo en que lo implementan en sus clases, se agrupan en los dos grandes núcleos temáticos resultantes tras el análisis de los datos, que ya hemos mencionado. Se han usado seudónimos para mantener el anonimato de los docentes. 


\subsection{Concepción del AC}

Todos los docentes participantes en el estudio de caso múltiple tienen conocimiento del trabajo de los principales referentes del AC, tanto a nivel general como en $\mathrm{EF}$, aunque varios de ellos manifiestan sus dudas a la hora de dar una definición precisa de lo que es el AC. Fernando lo reconoce abiertamente, "[...] después de años sigo teniendo algunas dudas con respecto a cómo delimitar el AC, se me siguen generando dudas", y Juan también manifiesta su inseguridad a la hora de delimitar qué es y qué no es $\mathrm{AC}$ :

La definición de AC siempre la he tenido un poco complicada, siempre se me ha resistido un poco. Me voy a las nociones básicas de lo que es la actividad cooperativa. Yo procuro que siempre haya una interrelación positiva entre las tareas de los alumnos y que las metas no sean incompatibles, sino que estén vinculadas. (JUAN, 2011)

En cualquier caso, todos los docentes distinguen perfectamente el AC de otros conceptos como el de trabajo en grupo o el de juego cooperativo. También coinciden en que para poder hablar de AC es necesaria la presencia de: (1) una interdependencia positiva de metas, (2) un trabajo en pequeños grupos, (3) un aprendizaje individual, y (4) una corresponsabilidad en el aprendizaje de todos y cada uno de los miembros del grupo. Carlos, por ejemplo, lo expone de la siguiente forma:

El AC sería un tipo de aprendizaje en grupo que tiene unas características muy determinadas: implica un trabajo en grupo en el que se da una interacción simultánea y una participación equitativa y además genera un aprendizaje individual de todas y cada una de las personas que componen el grupo. Implica esa doble responsabilidad de cada estudiante hacia su aprendizaje pero también hacia el de sus compañeros. El trabajo en grupo es simplemente pedir que se junten varias personas y realicen una determinada producción sin que se garanticen esas condiciones. (CARLOS, 2012) 


\subsection{Aplicación del AC}

Todos los docentes del estudio de caso múltiple manifiestan que el AC es uno de sus pilares metodológicos en sus programaciones de clase, aunque no todos los implementan con la misma frecuencia, ni de la misma manera. Para una mejor comprensión de los resultados, consideramos seis subcategorías de análisis: objetivos, contenidos, recursos didácticos, técnicas, grupos y evaluación, que desarrollamos a continuación.

\subsection{OBjetivos}

Hay un consenso entre los docentes a la hora de considerar que la práctica motriz es un medio ideal para el desarrollo de habilidades sociales y valores y, en consecuencia, las clases de EF deben convertirse en contextos que contribuyan a ello. En este sentido, todos los docentes están de acuerdo en que el AC es una de las estrategias metodológicas que promueven el logro afectivo y social, al tiempo que el alumnado alcanza unos niveles adecuados de aprendizaje motor. Juan, por ejemplo, manifiesta que:

Cuando trabajo con otra metodología quizá no puedo conseguir cosas que sí que me planteo con esta. Para mí, los objetivos globales de la EF, los aspectos centrales, son la experimentación de sensaciones motrices positivas, valoración de lo positivo que es la ayuda de los compañeros y de que tú ayudes a los demás, conseguir una mejora de habilidades sociales y habilidades emocionales, valorar positivamente el ejercicio físico como algo importante para la vida, para el tiempo de ocio. (JUAN, 2011)

\subsection{ConTENIDOS}

El análisis de las programaciones de los docentes nos muestra que mediante el trabajo con AC se desarrolla una amplia variedad de contenidos motores. Entre los contenidos trabajados por el profesorado con esta metodología destacan algunos con estructura 
ya de por sí cooperativa, como acrogimnasia, pero también otros de carácter individual, como es el caso de algunas propuestas de malabares. Incluso los docentes utilizan el AC para el trabajo de contenidos con un carácter marcadamente competitivo, como son los deportes de equipo. Antonio, por ejemplo, señala que introduce el AC en las unidades didácticas de: "[...] condición física, habilidades motrices, habilidades motrices básicas, bicicletas, habilidades gimnásticas y acrosport, expresión corporal y deportes". En el caso de contenidos con estructura competitiva, la tendencia de los docentes es la de combinar el trabajo de AC con otros modelos de enseñanza deportiva, fundamentalmente el modelo comprensivo.

\subsection{RECURSOS DIDÁCTICOS}

Los recursos didácticos utilizados por el profesorado cuando aplica el AC con su alumnado se orientan a facilitar a sus estudiantes la gestión autónoma de su proceso de aprendizaje en los diferentes grupos. Concretando aún más, los recursos que el docente proporciona a su alumnado tienen dos tipos de finalidades: (1) facilitar los procesos de evaluación entre los estudiantes, y (2) describir las tareas de aprendizaje que deben ser desarrolladas en los grupos. Carlos lo expresa claramente diciendo que le parece que los materiales didácticos "[...] facilitan que los grupos de AC puedan trabajar autónomamente. Ese es el principal objetivo. Ese y permitir evaluar el aprendizaje de los estudiantes". De este modo, los materiales más frecuentemente introducidos en las clases son instrumentos de autoevaluación y coevaluación, así como fichas de actividad que, en ocasiones, plantean problemas que deben ser resueltos por el alumnado y, en otras, además de describir la tarea que debe ser realizada, incluyen las claves para su aprendizaje en forma de frases cortas o de imágenes. Estos materiales forman parte del día a día de las sesiones de EF desarrolladas con AC, como lo demuestra, por ejemplo, el hecho de que durante el desarrollo de una clase de Andrés observamos cómo el docente se quejaba: "[...] solo un grupo me ha enseñado la hoja de evaluación", así que mientras "[...] algunos (estudiantes) siguen realizando acciones con el material, otros han ido a buscar sus hojas de evaluación y vuelven hasta el 
docente para enseñárselas"

\subsection{TÉCNICAS}

El profesorado participante conoce perfectamente las principales técnicas de AC y, muy especialmente, las más aplicadas en el área de EF. Sin embargo, ese conocimiento no se traduce en una implementación de dichas técnicas en sus clases o, al menos, no en todos los casos. Así, algunos docentes tienden a no aplicar las técnicas tal cual están descritas en la literatura, sino que parten de sus principios generales, pero las modifican en función de sus necesidades durante las sesiones. Este es el caso, por ejemplo, de Víctor, que expone:

Conozco algunas (técnicas) pero esa lectura que he ido haciendo de las diferentes propuestas, al final siempre la hago no para aplicarlas literalmente, sino para darle vueltas a ver cómo las voy adaptando a mi propuesta metodológica para que tenga sentido, es decir, no incorporo algo simplemente porque me parezca que en sí es bueno, sino porque creo que va a aportar algo a la propuesta que ya tengo estructurada. (CARLOS, 2012)

Fernando comenta que su "[...] planteamiento es de carácter más global, corresponsabilizar en el proceso de aprendizaje dentro del grupo y cumplir con todos los parámetros del AC". Por el contrario, otros docentes, como Antonio, explican que optan por una u otra técnica dependiendo "[...] del tipo de contenido o el tipo de tarea". En cualquier caso, hay algunas técnicas más habituales en las clases que otras. "Piensa - comparte - actúa" (GRINESKI, 1996, p. 30) es una de las más utilizadas. Juan, por ejemplo, comenta que trabaja "[...] mucho por desafíos o por retos [...]. Cualquier reto cooperativo en el que los alumnos tienen que compartir hipótesis de acción, tienen que hablar y tienen que escuchar y trabajar conjuntamente". Carlos comenta que las que más emplea fundamentalmente son "grupos de aprendizaje y piensa - comparte - actúa. Pero también enseñanza recíproca, yo hago - nosotros hacemos, descubrimiento compartido, tres vidas, el puzle de Aronso [...]". 


\subsection{GRuPOS}

Todos los docentes participantes están de acuerdo en trabajar mediante AC con grupos reducidos, de entre dos y ocho personas. Sin embargo, no hay unanimidad entre el profesorado en el modo de formar dichos grupos. Encontramos docentes que prefieren ser ellos quienes formen los equipos de aprendizaje, con el fin de garantizar su heterogeneidad, como dice Laura, "[...] para no hacer guetos, pero también porque la diversidad es enriquecedora y porque la vida misma es muy heterogénea en todos los sentidos". Otros, como Juan, dejan libertad a su alumnado para agruparse "[...]bajo las premisas que yo les mando. No he necesitado prácticamente nunca cambiar los grupos u obligarles a mezclarse, salvo los típicos problemas de inicio de unidad didáctica o de inicio de curso". En cualquier caso, todo parece indicar que los docentes no siguen unos criterios rígidos, de modo que es bastante habitual alternar diferentes procesos para la formación de grupos. Así, por ejemplo, Antonio deja a sus estudiantes elegir compañero a la hora de "[...] trabajar por parejas, con la condición de que sean mixtas", pero prefiere formarlos él cuando se trata de grupos de mayor tamaño o "[...]si el trabajo con el modelo cooperativo va a durar varios días y se van a mantener los grupos", ya que "[...]no puedes arriesgarte a que los grupos no estén bien configurados".

\subsection{EVALUACIÓN}

Todo el profesorado participante subraya la importancia de la evaluación en los procesos de AC y destaca que un elemento clave de la eficacia de esta metodología radica en que el docente y el alumnado puedan comprobar cuál ha sido el resultado de todo el proceso de aprendizaje. También hay unanimidad entre los docentes a la hora de considerar que deben ser evaluados no solo los logros motores del alumnado, sino también los aspectos sociales y el proceso de aprendizaje entre iguales a través del cual han sido alcanzados. Esto incluye la evaluación de los elementos cognitivos, actitudinales y afectivos que inciden en los procesos de toma de decisiones en los diferentes grupos. Fernando lo explica al referirse a una unidad didáctica de voleibol: 
Cada alumno tiene que valorar al final: ¿qué progresos he vivido en la capacidad para tomar decisiones?, ¿qué procesos he vivido en las habilidades técnicas, en el golpe de antebrazos, en el toque de dedos, el saque y el remate? Y lo planteamos así, en evolución o en progresos; y las claves son de progresos. (FERNANDO, 2011)

Desde estas premisas, el profesorado introduce múltiples y variados procedimientos para la evaluación. Carlos comenta: "[...] la evaluación me parece importante y por ello intento que la información me llegue por varios canales, de ahí que use diferentes instrumentos y además variados, para triangular los datos". Entre los más habituales destacan algunos no estructurados, como las asambleas o las tutorías individuales; y otros estructurados, como listas de control o diferentes fichas de autoevaluación y de coevaluación.

La evaluación del componente social del aprendizaje es especialmente importante para el profesorado que implementa el AC en sus clases. Así, algunos docentes, como Laura, piden "[...] un diario de conflictos para que aparezcan los conflictos y la manera de resolverlos. Si se resolvieron, si no, si tuvieron que acudir a mí [...]". Antonio utiliza el "registro de anécdotas" donde hace "anotaciones en positivo o en negativo [...] sobre aspectos vinculados a las ayudas o a la colaboración dentro del grupo".

Los docentes que aplican el AC en sus clases apuestan por una evaluación formativa y compartida entre docente y estudiantes, centrada, como dice Carlos, "[...] no solo en los resultados, sino también en el proceso que se desarrolla en los grupos". El docente contrasta sus valoraciones con las de su alumnado y establece procesos dialogados de consenso orientados a favorecer el aprendizaje. Víctor, por ejemplo, lo explica de la siguiente manera:

Puedo tener registros de comportamiento individual o del grupo de trabajo, que debería de estar en consonancia con la autoevaluación que ha hecho ese grupo. Si no coincide lo que ellos exponen con lo que yo he observado, al día siguiente aprovecho 
para hacer un comentario al inicio del trabajo grupal. Me acerco a ese grupo y hago ese proceso de evaluación en el que vemos si realmente están ayudándose entre sí, si se está produciendo una interdependencia positiva... Es precisamente en esos procesos de obtención de información del día a día donde se va viendo si realmente su trabajo se está vinculando entre ellos, si están aportando o no están trabajando cooperativamente para el objetivo común. (VICTOR, 2011)

\section{DISCUSIÓN Y CONCLUSIONES}

Todos los docentes participantes en el estudio de caso múltiple conocen los principios generales del AC. Así, identifican como características de esta metodología: la interdependencia positiva de metas, el trabajo grupal, el aprendizaje individual y la corresponsabilidad en el aprendizaje de todos y cada uno de los miembros del grupo. Todo ello estaría de acuerdo con las definiciones expuestas en la literatura por los principales referentes del AC, tanto a nivel general (JOHNSON; JOHNSON, 1999, 2009; KAGAN, 2000; PUJOLÀS, 2008; SLAVIN, 1999), como en EF (DYSON; CASEY, 2012; METZLER, 2011; VELÁZQUEZ, 2010).

Los docentes que introducen el $\mathrm{AC}$ en sus clases de $\mathrm{EF}$ lo conciben como una importante estrategia metodológica que, además del aprendizaje motor, permite a los estudiantes alcanzar objetivos sociales y afectivo-motivacionales. Diferentes investigaciones en el ámbito motor refuerzan esta idea (BÄHR, 2010; BÄHR; WIBOWO, 2012; DYSON, 2001) y apuntan al AC como una estrategia inclusiva en las clases de EF (DOWLER, 2012; GRENIER; YEATON, 2012; VELÁZQUEZ, 2012a).

Cualquier contenido puede ser enseñado con AC (JOHNSON; JOHNSON, 1999; PUTNAM, 1997). Esta idea se concreta en el ámbito motor, de modo que un simple análisis de la literatura nos muestra la posibilidad de trabajar una gran diversidad de contenidos motores mediante AC, desde habilidades gimnásticas (ANDRÉ et 
al. 2011; B ̈̈HR, 2010; BARBA, 2010) a los deportes individuales (CASEY, 2010) y colectivos (DYSON, 2001). Los docentes entienden que la forma de estructurar el proceso de aprendizaje es independiente de la estructura de la tarea que debe ser enseñada. Así, aplican el AC en sus clases no solo para promover el aprendizaje de tareas individuales o cooperativas, sino también para el trabajo de contenidos de carácter competitivo.

El AC conlleva un contexto de aprendizaje basado en la interacción entre iguales, con el que el alumnado puede estar poco familiarizado. Con el fin de facilitar a sus estudiantes el proceso de gestión autónoma de su trabajo grupal, los docentes participantes utilizan diferentes recursos didácticos. En ocasiones, estos recursos describen las tareas que van a ser desarrolladas en los grupos, subrayando sus claves de aprendizaje (CASEY, 2010; VELÁZQUEZ, 2010). Otras veces se orientan a facilitar los procesos de coevaluación entre los estudiantes (FERNÁNDEZ-RÍO, 2011; VELÁZQUEZ, 2012b).

Aunque en la literatura es posible encontrar descripciones precisas de cómo aplicar diferentes técnicas estructuradas de AC en EF (GRINESKI, 1996; VELÁZQUEZ, 2004), la mayoría de los docentes de EF no utilizan estas técnicas en sus clases. Por el contrario, tienden a introducir trabajos en grupo en los que el docente tiende a reforzar diferentes conductas prosociales que pretende que se manifiesten durante las tareas. En otras ocasiones, los docentes parten de una técnica concreta pero modifican su estructura para adaptarla a las necesidades específicas de su alumnado.

La práctica totalidad de los referentes sobre AC apuestan por la máxima heterogeneidad en los grupos (COHEN, 1999; JOHNSON; JOHNSON; HOLUBEC, 1999; PUJOLÀS, 2008; SLAVIN, 1999), algo que, también es aceptado por el profesorado estudiado. Sin embargo, encontramos divergencia entre las fórmulas más aconsejadas a nivel teórico para constituir los agrupamientos y el modo en que los docentes plantean la formación de los grupos en sus clases. Así, mientras en la literatura se aconseja que sea el docente quien, de un modo total o parcial, controle el proceso de constitución 
de los equipos de aprendizaje (COHEN, 1999; JOHNSON; JOHNSON, 1999; METZLER, 2011), el profesorado parece buscar una especie de equilibrio entre los deseos de su alumnado de agruparse por afinidad y el interés del docente por proporcionar a sus estudiantes la oportunidad de trabajar con compañeros que inicialmente no elegirían. De este modo, la manera más habitual de agrupar al alumnado consiste en permitir a los estudiantes elegir a sus compañeros de equipo, siempre que este cumpla unas condiciones, impuestas por el docente, que garanticen la heterogeneidad de los grupos formados.

Todos los docentes participantes valoran la importancia de la evaluación en los procesos de AC. Algunos destacan incluso que un elemento clave de la eficacia de esta metodología radica en que el docente y el alumnado puedan comprobar cuál ha sido el resultado de todo el proceso de aprendizaje. También hay unanimidad a la hora de considerar que deben ser evaluados no solo los logros motores y sociales del alumnado, sino también el proceso de aprendizaje entre iguales a través del cual han sido alcanzados. Esto incluye la evaluación de los elementos cognitivos, actitudinales y afectivos que inciden en los procesos de toma de decisiones en los diferentes grupos. Desde estas premisas, el profesorado introduce diferentes procedimientos para la evaluación, algunos no estructurados, como las reflexiones al final de las sesiones; otros estructurados, como la observación registrada a través de planillas de control o de planillas de autoevaluación y coevaluación del alumnado. El análisis de la literatura nos permite comprobar que la evaluación asociada al AC se caracteriza por: (1) estar integrada en el propio proceso de aprendizaje grupal (CASEY, 2010; JOHNSON; JOHNSON, 199; JOHNSON; JOHNSON; HOLUBEC, 1999; VELÁZQUEZ, 2010), (2) incluir procesos de autoevaluación y coevaluación (FERNÁNDEZ-RÍO, 2011; FRAILE, 2012; LÓPEZ PASTOR et al., 2010), (3) utilizar múltiples y variados instrumentos (JOHNSON; JOHNSON, 1999; LÓPEZ-PASTOR et al., 2010; PUJOLÀS, 2008; VELÁZQUEZ, 2012b), y (4) orientarse a valorar logros motores, sociales y actitudinales cooperativo (JOHNSON; JOHNSON, 2004; LÓPEZ-PASTOR et al., 2010; VELÁZQUEZ, 2012b). Estas 
características de la evaluación, reflejadas en la literatura, son coincidentes con las encontradas en el estudio del profesorado que aplica el AC en sus clases de EF.

En este trabajo hemos analizado la concepción que tienen del $\mathrm{AC}$ un grupo de docentes de Educación Física españoles que son reconocidos como referentes en esta metodología, así como las características más importantes de su forma de llevarlo a cabo en el aula. Consideramos que los resultados encontrados pueden ser de utilidad al profesorado de EF interesado en desarrollar procesos de AC con su alumnado, así como a los investigadores especializados en esta temática. 


\section{Cooperative learning in Physical Education}

Abstract: The purpose of the study is to know and analyze the concept of cooperative learning (CL) of a group of Spanish teachers of Physical Education who are recognized as references in this methodology, as well as the way they implement it in the classroom. It is a multiple case study that utilized interviews, not participant observation and documental analysis. The results indicate that all the participant teachers know the general principles of $\mathrm{CL}$, utilize and consider them as an important methodology to develop the different aspects of learning. Besides, the teachers consider that any content can be taught using the $C L$ and value the importance of the formative and shared evaluation. Keywords: learning. Evaluation. Qualitative research.

\footnotetext{
Aprendizagem cooperativa na Educação Física Resumo: A finalidade do estudo é conhecer e analisar a concepção de aprendizagem cooperativa (AC) de um grupo de docentes espanhóis de Educação Física, reconhecidos como referência nesta metodologia, assim como o modo que adotam para implementá-la em suas aulas. Trata-se de um estudo de caso múltiplo que utilizou entrevistas, observação não participante e análise documental. Os resultados indicam que todos os docentes participantes conhecem os princípios gerais da AC e os utilizam, concebendo-os como uma importante metodologia para avançar nos diferentes âmbitos da aprendizagem. Além disso, consideram que qualquer conteúdo pode ser ensinado por meio da AC e valorizam a importância da avaliação formativa e compartilhada.

Palavras-chave: aprendizagem. Avaliação. Pesquisa qualitativa.
} 


\section{REFERENCIAS}

ANDRÉ, Amael. Influence de l'Apprentissage Coopératif sur le savoir s'échauffer et la motivation autodéterminée vis à vis de l'échauffement. eJRIEPS, v. 27, p. 5-26, 2012. Disponible en: <http://www.fcomte.iufm.fr/ejrieps>. Acceso en: 24 de nov 2012.

ANDRÉ, Amael; DENEUVE, Pascale; LOUVET, Benoit. Cooperative Learning in Physical Education and acceptance of students with learning disabilities. Journal of Applied Sport Psychology,Abingdon, v. 23, n.4, p. 474-485, out./dez. 2011. doi: 10.1080/10413200.2011.580826.

BÄHR, Ingrid. Experiencia práctica y resultados empíricos sobre el aprendizaje cooperativo en gimnasia. In: VELÁZQUEZ, Carlos (Coord.). Aprendizaje cooperativo en Educación Física. Fundamentos y aplicaciones prácticas. Barcelona: INDE, 2010. p. 149-163.

BÄHR, Ingrid.; WIBOWO, Jonas. Teacher action in the Cooperative Learning model in the physical education classroom. In: DYSON, Ben; CASEY, Ashley (Eds.). Cooperative learning in Physical Education. A research-based approach. London: Routledge, 2012. p. 27-41.

BARBA, José J. Diferencias entre el aprendizaje cooperativo y la asignación de tareas en la Escuela Rural. Comparación de dos estudios de caso en una unidad didáctica de acrosport en segundo ciclo de primaria. Retos: nuevas tendencias en educación física, deportes y recreación, San Javier, n. 18, p. 14-18, jul. 2010.

BARRETT, Tim. Effects of cooperative learning on the performance of sixth-grade Physical Education students. Journal of Teaching in Physical Education, Champaign, v. 24, n.1, p. 88-102, jan./mar. 2005.

CASEY, Ashley. El aprendizaje cooperativo aplicado a la enseñanza del atletismo en la escuela secundaria. In: Velázquez, Carlos (Coord.). Aprendizaje cooperativo en Educación Física. Fundamentos y aplicaciones prácticas. Barcelona: INDE, 2010. p. 187-199.

CASEY, Ashley. Cooperative Learning through the eyes of a teacher-researcher and his students. In: B. Dyson; A. Casey (Eds.). Cooperative learning in Physical Education. A research-based approach. London: Routledge, 2012. p. 75-87.

COHEN, Elizabeth G. Organizzare i gruppi cooperativi. Ruoli, funzioni, attività. Gardolo, TN: Erickson, 1999.

COHEN, Elizabeth G.; LOTAN, Rachel A.; LEECHOR, Chaub. Can classrooms learn? Sociology of Education, Washington, v. 62, n. 2, p. 75-94, abr. 1989 doi: 10.2307/ 2112841

DENZIN, Norman K.; LINCOLN, Yvonna S. (Eds.). The SAGE handbook of qualitative research. Thousand Oaks, CA: Sage, 2005. 
DOWLER, Wendy. Cooperative Learning and interactions in inclusive secondaryschool physical education classes in Australia. In: DYSON, Ben; CASEY, Ashley (Eds.). Cooperative learning in Physical Education. A research-based approach. London: Routledge, 2012. p. 150-165.

DYSON, Ben. Cooperative learning in an elementary Physical Education program. Journal of Teaching in Physical Education, Champaign, vol. 20, n. 3, p. 264281, jul./set. 2001.

DYSON, Ben; CASEY, Ashley (Eds.). Cooperative learning in Physical Education. A research-based approach. London: Routledge, 2012.

FATHMAN, Ann K.; KESSLER, Carolyn. Cooperative language learning in school contexts. Annual Review of Applied Linguistics, New York, n. 13, p. 127-140, 1992. doi: $10.1017 /$ S0267190500002439

FERNA?NDEZ-RI?O, Javier. El aprendizaje cooperativo en el aula de educación física para la integración en el medio social: análisis comparativo con otros sistemas de enseñanza y aprendizaje. [CD-ROM]. Valladolid: La Peonza, 2003.

FERNÁNDEZ-RÍO, Javier. La enseñanza del bádminton a través de la hibridación de los modelos de aprendizaje cooperativo, táctico y educación deportiva y del uso de materiales autoconstruidos. In: Méndez, Antonio (Coord.). Modelos actuales de iniciación deportiva. Unidades didácticas sobre juegos y deportes de cancha dividida. Sevilla: Wanceulen, 2011. p. 193-236.

FRAILE, Antonio. Evaluación formativa e interdisciplinariedad: análisis de dos asignaturas con el mismo sistema de evaluación. Psychology, Society, \& Education, San Sebastián de los Reyes, vol. 4, n. 1, p. 5-16, mai. 2012.

GILLIES, Robyn M. Teachers' and students' verbal behaviours during cooperative and small-group learning. British Journal of Educational Psychology, Hoboken, vol. 76, n. 2, p. 271-287, jun. 2006. doi: 10.1007/978-0-387-70892-8_12.

GOUDAS, Marios; MAGOTSIOU, Evmorfia. The effects of a cooperative Physical Education program on students' social skills. Journal of Applied Sport Psychology, Abingdon, vol. 21, n. 3, p. 356-364, jul. 2009. doi: 10.1080/ 10413200903026058.

GRENIER, Michelle; DYSON, Ben; YEATON, Pat. Cooperative learning that includes students with disabilities. Journal of Physical Education, Recreation and Dance, Reston, vol. 76, n. 6, p. 29-35, ago. 2005.

GRENIER, Michelle; Yeaton, Pat. The Cooperative Learning model as an inclusive pedagogical practice in physical education. In: B. Dyson; A. Casey (Eds.). Cooperative learning in Physical Education. A research-based approach. London: Routledge, 2012. p. 119-135.

GRINESKI, Steve. Cooperative learning in Physical Education. Champaign, IL: Human Kinetics, 1996. 
GUBA, Egon G. Criterios de credibilidad en la investigación naturalista. In: GIMENO, José; PÉREZ GÓMEZ, Ángel (Eds.). La enseñanza: su teoría y su práctica. Madrid: Akal, 1989. p. 148-165.

MILES, Matthew. B.; HUBERMAN, A. Michael. Qualitative data analysis: An expanded sourcebook. Thousand Oaks, CA: Sage, 1994.

JOHNSON, David W.; JOHNSON, Roger T. Aprender juntos y solos. Aprendizaje cooperativo, competitivo e individualista. Buenos Aires: Aique, 1999.

JOHNSON, David W.; JOHNSON, Roger T. Assessing students in groups. Promoting group responsibility and individual accountability. Thousand Oaks, CA: SAGE, 2004.

JOHNSON, David W.; JOHNSON, Roger T. An educational psychology success story: social interdependence theory and cooperative learning. Educational Researcher, Thousand Oaks, vol. 38, n. 5, p. 365-379, jun. 2009. doi: 10.3102/ $0013189 \times 09339057$.

JOHNSON, David W.; JOHNSON, Roger T.; HOLUBEC, Edithe J. Los nuevos círculos del aprendizaje. La cooperación en el aula y la escuela. Buenos Aires: Aique, 1999.

KAGAN, Spencer. L'apprendimento cooperativo: I'approccio strutturale. Roma: Edizioni Lavoro, 2000.

LÓPEZ PASTOR, Víctor M.; BARBA, José J.; VACAS, Roberto A.; Gonzalo, L. Alberto. La evaluación en educación física y las actividades físicas cooperativas. ¿Somos coherentes? Las posibilidades de la evaluación formativa y compartida. In: VELÁZQUEZ, Carlos (Coord.). Aprendizaje cooperativo en Educación Física. Fundamentos y aplicaciones prácticas. Barcelona: INDE, 2010, p. 225-255.

MERTON, Robert K.; FISKE, Marjorie; KENDALL, Patricia L. Propósitos y criterios de la entrevista focalizada. Empiria, Madrid, n. 1, 1989, p. 215-227.

METZLER, Michael W. Instructional models for Physical Education. Scottsdale, AZ: Holcomb Hathaway, 2011.

POLVI, Singa; TELAMA, Risto. The use of cooperative learning as a social enhancer in Physical Education. Scandinavian Journal of Educational Research, Abingdon, vol. 44, n. 1, p. 105-115, jan. 2000. doi: 10.1080/713696660.

PUJOLÀS, Pere. El aprendizaje cooperativo. 9 ideas clave. Barcelona: Graó, 2008.

PUTNAM, JoAnne. Cooperative learning in diverse classrooms. Upper Saddle River, N.J. Prentice-Hall, 1997.

RUIZ-OLABUÉNAGA, J. Ignacio. Metodología de la investigacion cualitativa. Bilbao: Universidad de Deusto, 2012. 
SLAVIN, Robert E. Aprendizaje cooperativo. Teoría, investigación y práctica. Buenos Aires: Aique, 1999.

VELÁZQUEZ, Carlos. Las actividades físicas cooperativas. Una propuesta para la formación de valores a través de la educación física en las escuelas de educación básica. México, D.F.: Secretaría de Educación Pública, 2004.

VELÁZQUEZ, Carlos (Coord.). Aprendizaje cooperativo en Educación Física. Fundamentos y aplicaciones prácticas. Barcelona: INDE, 2010.

VELÁZQUEZ, C. Analysis of the effects of the implementation of cooperative learning in Physical Education. Qualitative research in education, Barcelona, n. 1, vol. 1, p. 80-105, jun. 2010a. doi: 10.4471/qre.2012.04.

VELÁZQUEZ, C. Putting cooperative learning and physical activity into practice with primary students. In: DYSON, Ben; CASEY, Ashley (Eds.). Cooperative learning in Physical Education. A research-based approach. London: Routledge, 2012b. p. 59-74.

Endereço para correspondência:

Carlos Velázquez Callado

Facultad de Educación y Trabajo Social

Departamento de Didáctica de la Expresión Musical, Plástica y Corporal

Paseo de Belén, 1

47011 - Valladolid

(España)

Recebido em: 17.06.2013

Aprovado em: 11.12.2013

Vovimento, Porto Alegre, v. 20, n. 01, p. 239-259, jan/mar de 2014. 